
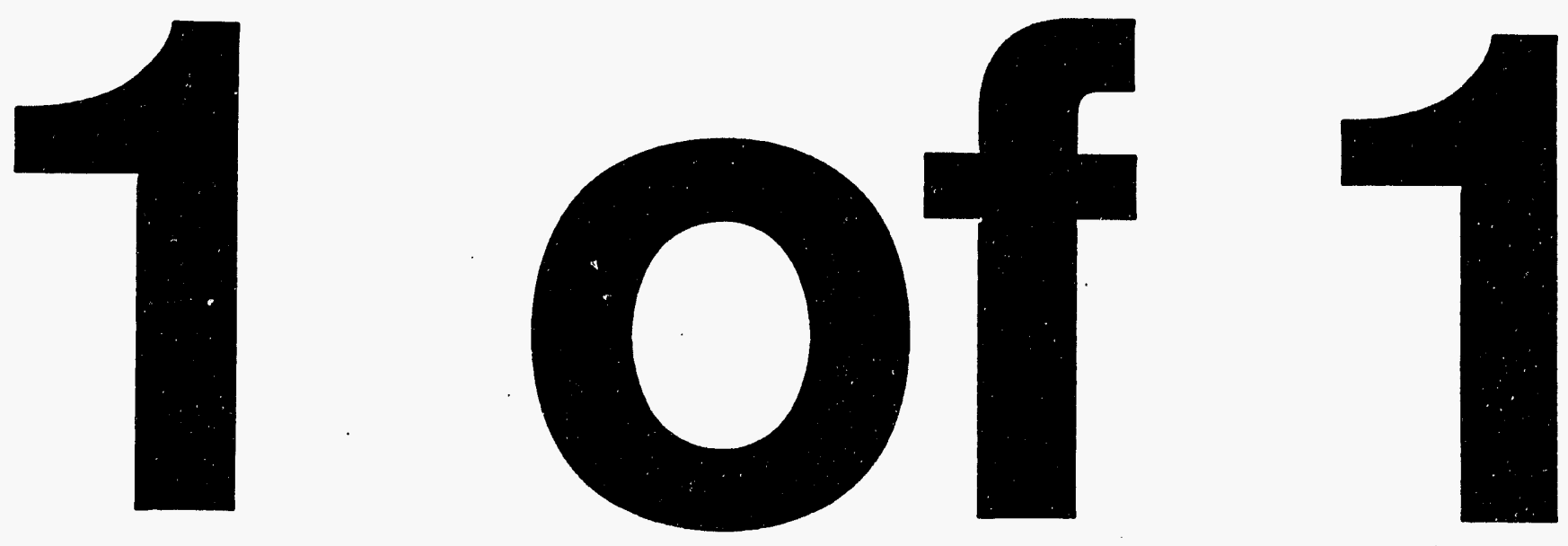


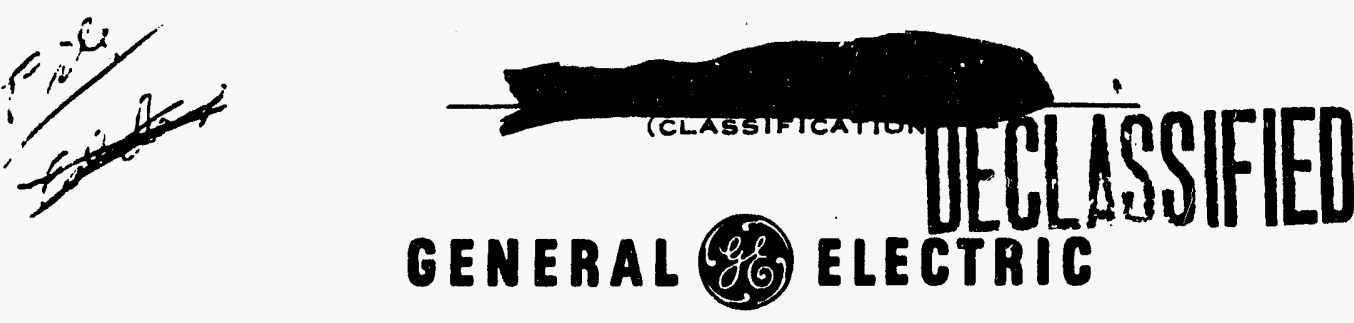

HANFORD ATOMIC PRODUCTS ORERATION - RICHLAND, WASHINGTON

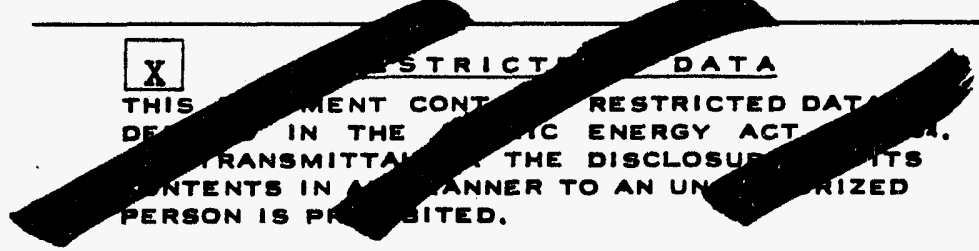

TITLE

OLD PIIE OPERATION WITH VARYING

AMOUNISS OF E-METAL

HW-69550 RD

EERIES ANO COPY NO.

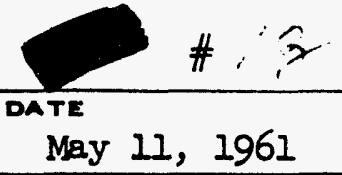

May 11, 1961

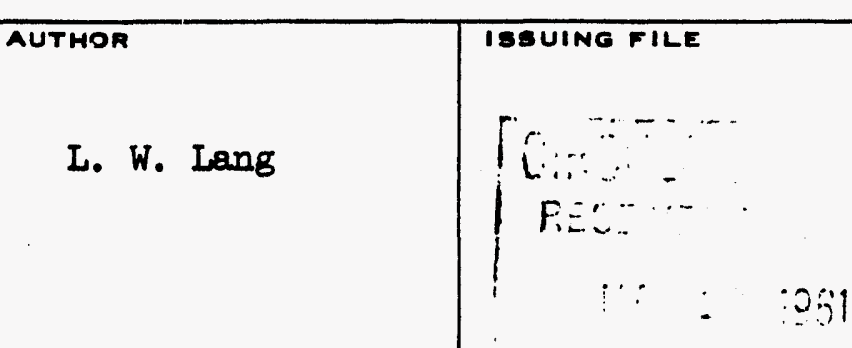

OTHER OFFICIAL CLABBIFIED INFORMATION

THIS MATERIAL CONTAINS INFORMATION AFFECTING THE NATIONAL DEFENSE OF THE UNITED STATES WITHIN THE MEANING OF THE ESPIONAGE LAWS. TITLE 18, U.S.C., SECS. T93 AND 794, THE TRANSMISSION OR REVELATION OF WHICH IN ANY MANNER TO AN UNAUTHORIZED PERBON IS PROHIBITED BY LAW.

THIS DOCUMEN TOT DE LEFT UNATT WHERE AN UNAUTMORIZED TO IT. WHF IN USE, IT MUST DE ? AN AN APPROVED LOCKED RT GUARD? WHILE IT IS YOUP WSBION AND UNTIL YOU HAM CLPAILES, IT IS YOU ONSIBILITY TO KEEP IT TROJECT AND FROM AM

UROJECT AND FROM ANTHORIZED PERSON. ITS TS TTAL TO, AND STORAG

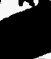

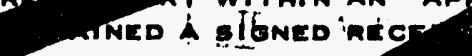
CONTENTS WITHIN TH

GTESIDENCE IS PROH THOTT IS NOT TO DE DU TO SION IN THE BR MOVIDED BELOW.

\begin{tabular}{c|c|c|c|c|} 
TO SION IN THE BR & FILESIDED BELOW. & ROUTE & SIGNATURE AND DATE \\
\hline ROUTE TO: & PAYROLL NO. & LOCATION & DATE. \\
\hline
\end{tabular}

54-3000-340 (3-57) AEC.GE MICHLAND. WAEH

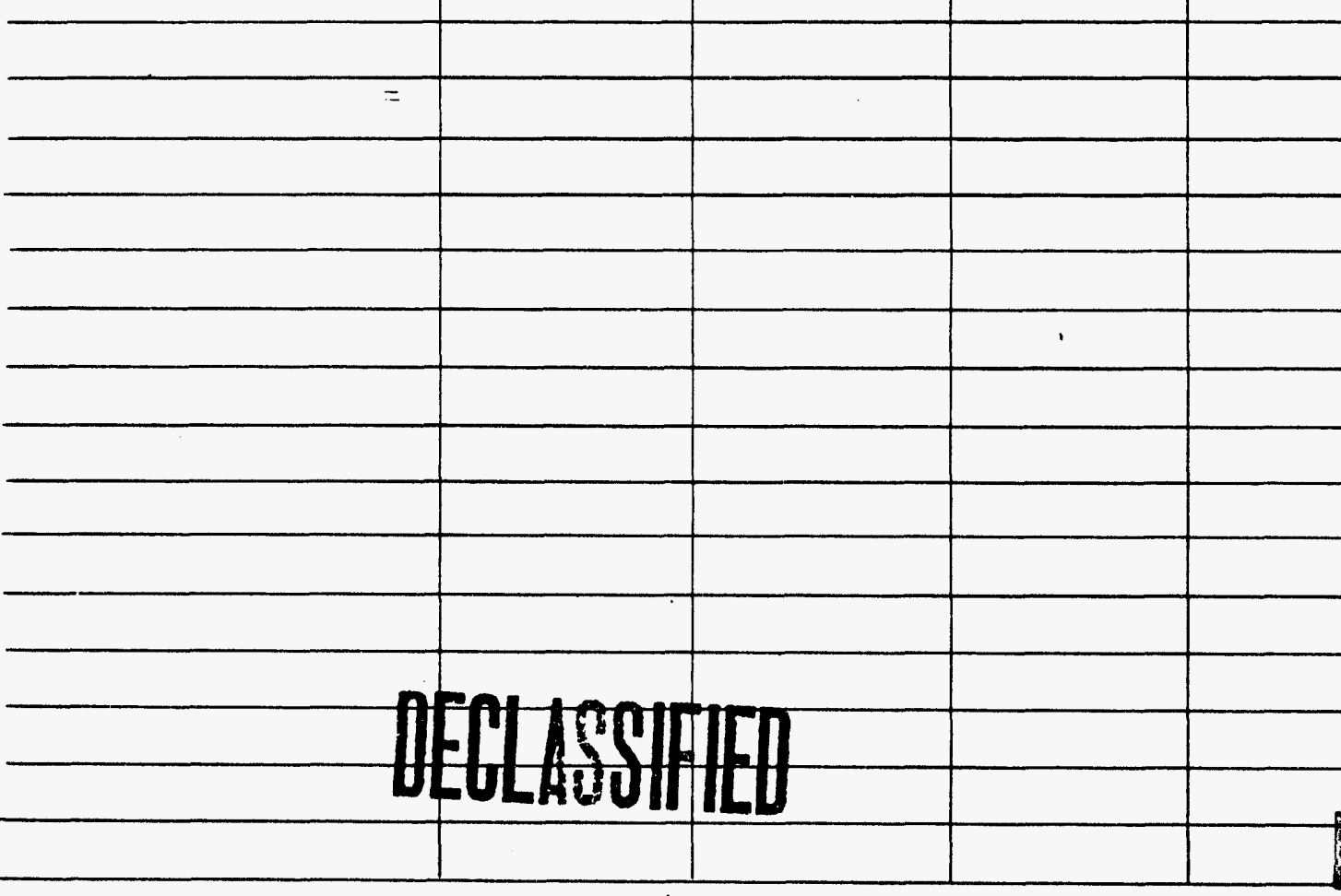

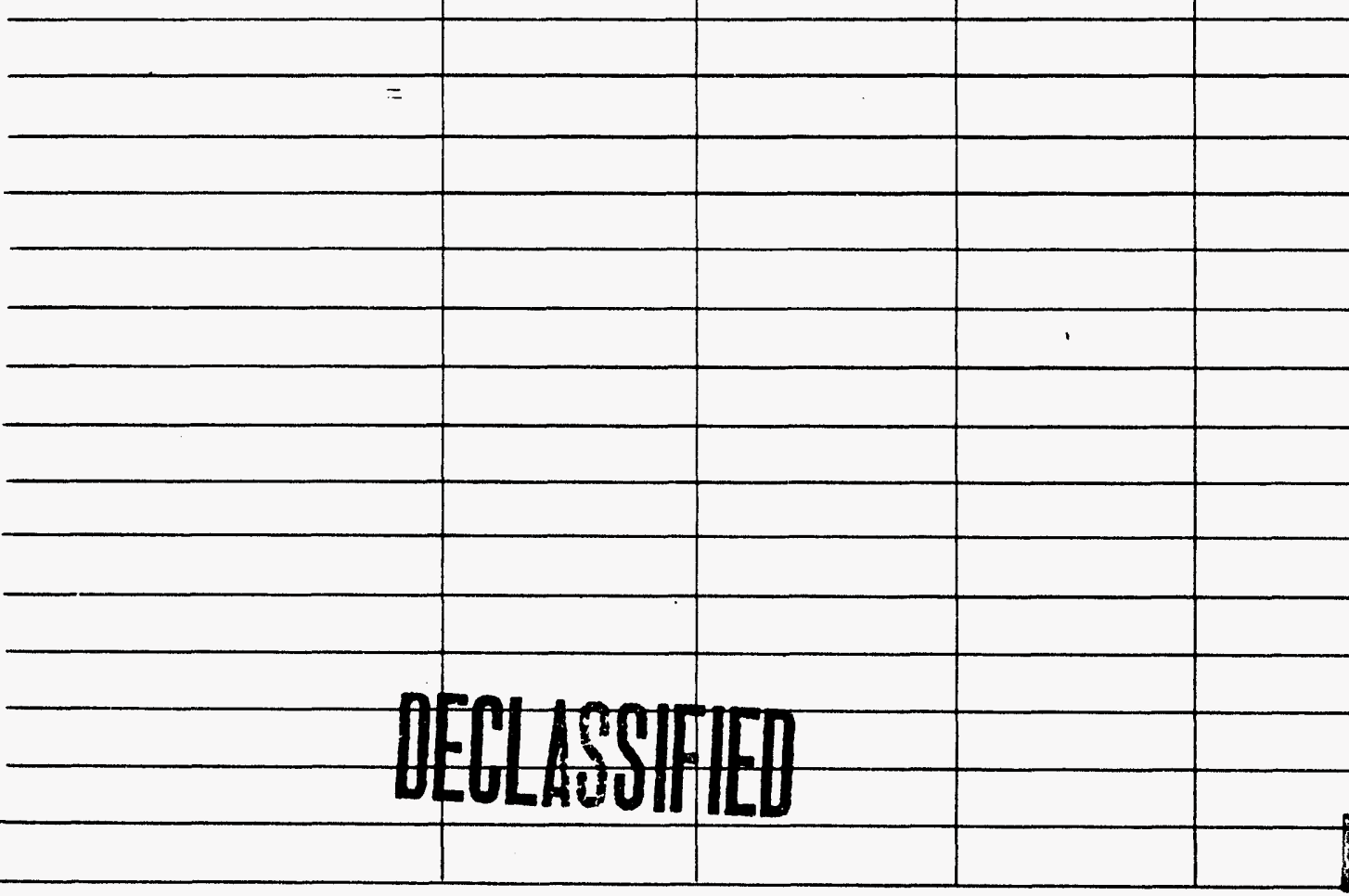
MASTER

(CLASSIFTCATIOON) 
Distribution

\#1-13. LW Lang
This document consists of pages. Copy No. $1 \frac{3}{2}$ of coples.

$\frac{2}{13}$

\section{OLD PILE OPERATION WITH VARYINTG AMOUNTS OF E-METAL}

by

L. W. LANG

May 11, 1961
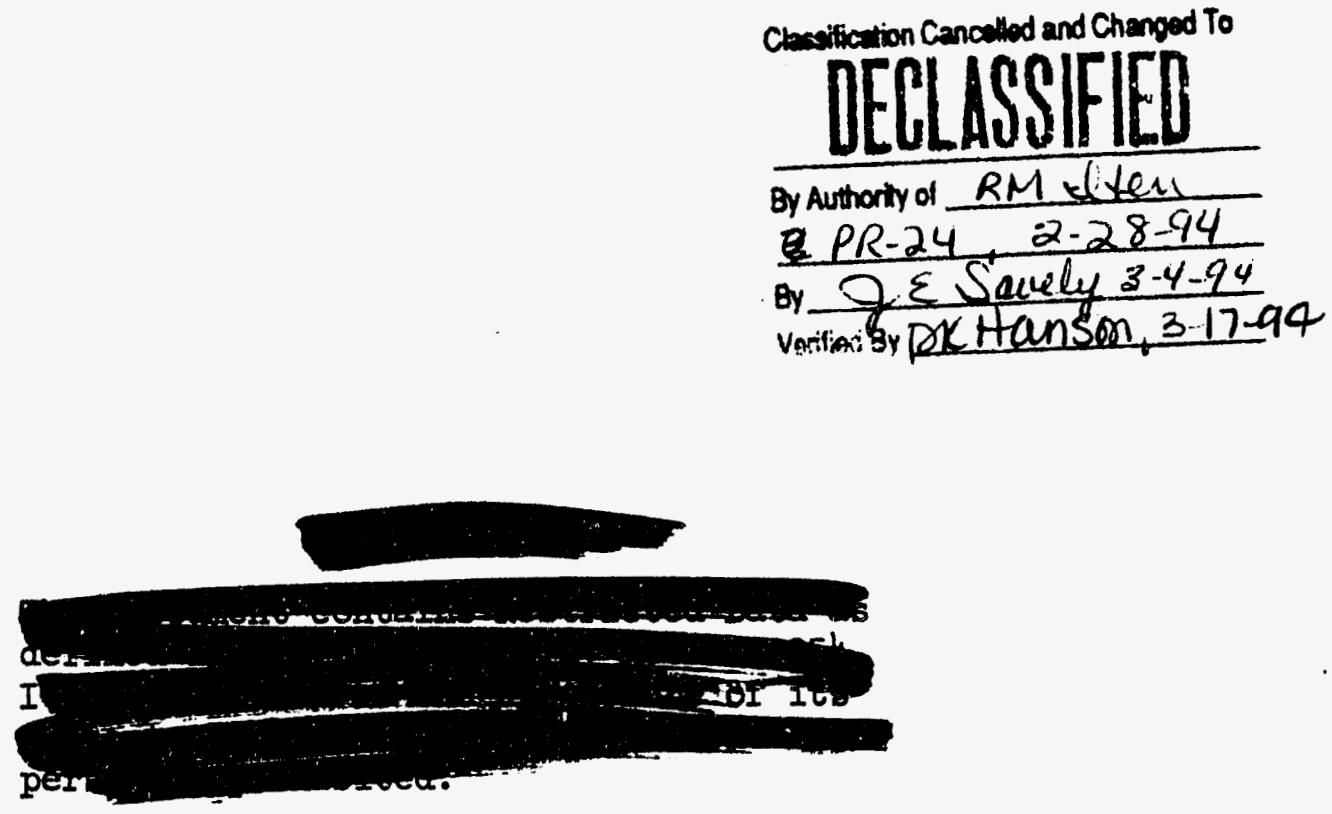
OLD PILE OPERATION WITH VARYING AMOUNTS OF E-METAL

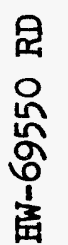

\% of Pile Power

Exposure

Conversion Ratio

$\mathrm{Pu}$

Tritium (Pu Equiv/MWD)

\% 240

Over-All Conversion

$\mathrm{Pu}$ (g/MWD)

Tritium ( $P u$ equiv/MWD) TOTAL

\% 240

\% Increase Conv. Ratios

Combined Operation $\%$

Increase (SR Makes

$.889 \mathrm{~g}$ Pu/equiv $\mathrm{T}_{2}$ )

Over-All Efficiency

(\% Change)

Miscellaneous Information

K-Piles Conversion Ratio Increase

Total Tritium from Blanket

$\mathrm{E}-\mathrm{N}$

GVR Ratio (Total Gas) E-N

\footnotetext{
Blanket
@ $1-1 / 2$ y

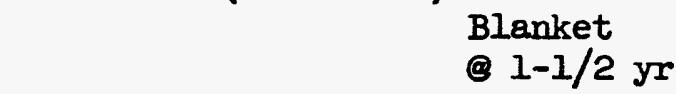

\section{Present Operation}

Nat

.947

$\frac{\text { Blanket }}{\text { Nat } .947}$

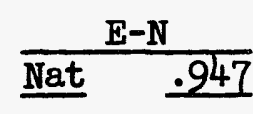

6

\begin{tabular}{lllll} 
& & & & Overbore Case \\
\hline \multicolumn{2}{c}{ Base } & & \multicolumn{1}{l}{ With Blanket } \\
\hline Nat & .947 & & Nat & .947 \\
67.6 & 32.4 & 49.1 & 50.9 \\
700 & 800 & 700 & 800 \\
& & & \\
.998 & .873 & 1.008 & .884 \\
6.15 & 5.45 & 6.15 & 5.45
\end{tabular}

.758

5.45

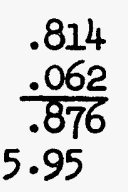

4.8

3.9

$-18$

$$
\begin{array}{r}
.758 \\
.202 \\
\hline .960
\end{array}
$$$$
5.45
$$

14.8

12.2

$-2 \%$

.957
$\frac{.957}{5.94}$
14.6

14.6

$+2 \%$

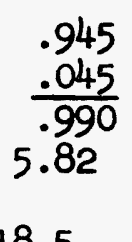

18.5

17.8

$+1 \%$

$\frac{\text { With E-N }}{\text { Nat } \quad .947}$

o 100

800

916 .146 5.45
3. 3\% for Blanket $9.8 \%$ for $\mathrm{E}-\mathrm{N}$$$
4.0 \mathrm{~kg}
$$$$
13.0
$$$$
\begin{array}{r}
13.0 \\
\hline
\end{array}
$$$$
6-12.0
$$

Reference: HW-68697, "Comparative Return for Overbore and E-N Overbore and E-N Loadings, B, D, F and DR Reactors," Nechodom, W. S. (3-22-61). 

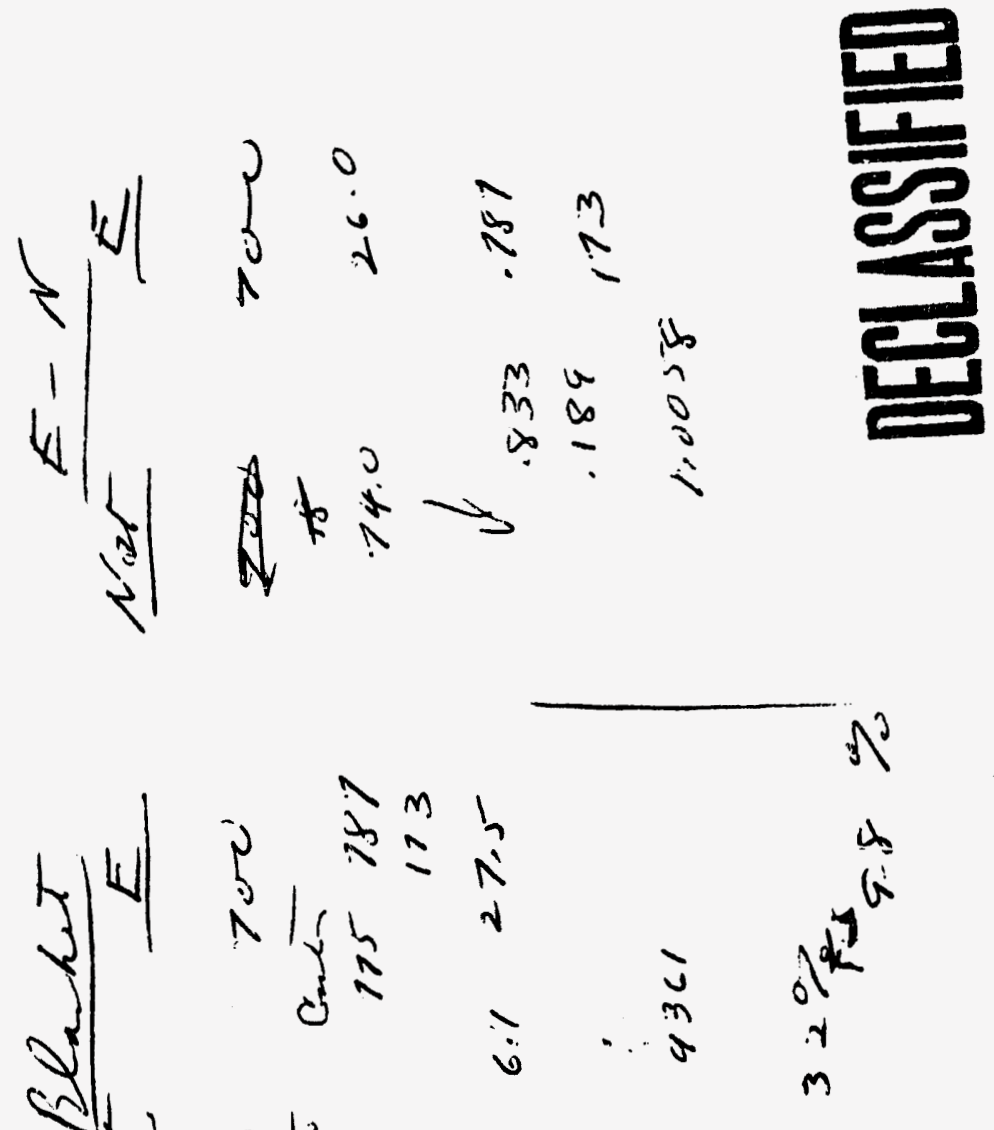

जilk

$\frac{\sqrt{3}}{3}$

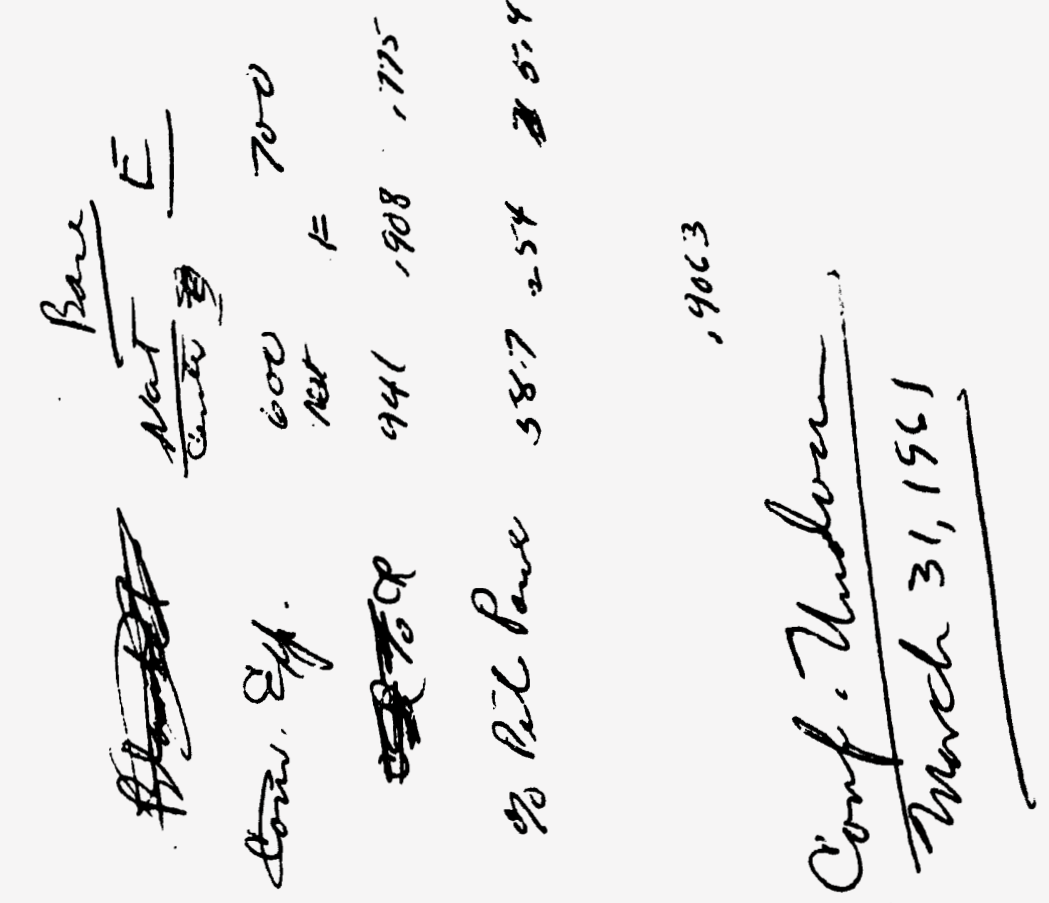

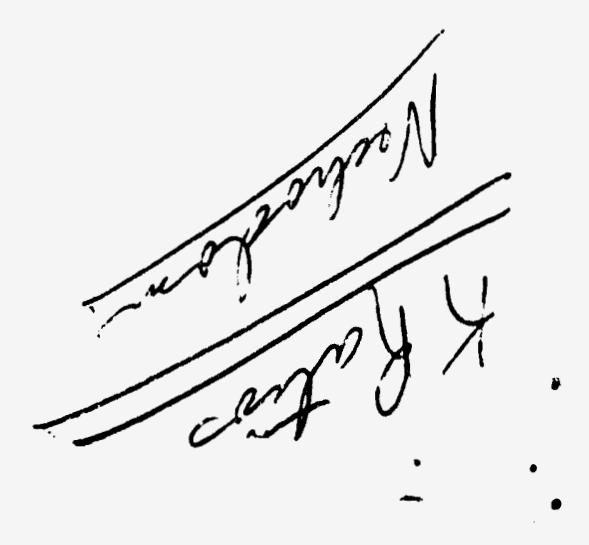


Buse

Calculate (

leng. $856 \times 62.6 \quad 536$

$$
.825-x 25.7 \frac{24.5}{78.1 / 52,3}=, 840 \times 5.8
$$

E-NCire+ Haskd

$$
\begin{array}{ll}
.772 \times 695 & P_{11} \\
.206 & .5365 \\
.726 \times 305 & \frac{2214}{1432} \\
.193 & \frac{584}{7579} \frac{2021}{278}
\end{array}
$$

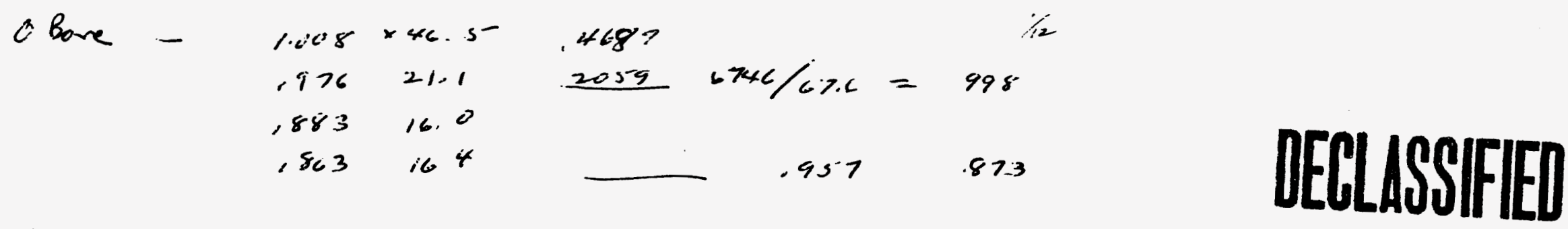

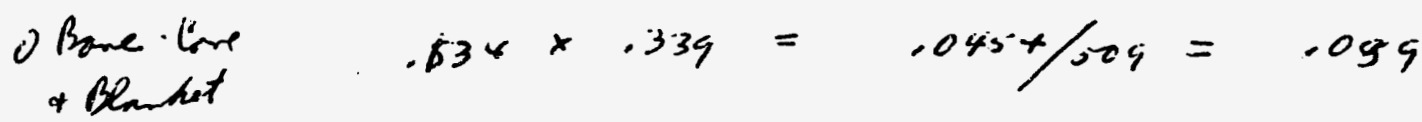

$$
\begin{aligned}
& E-N \\
& .931 \times 16>6 \\
& \text { ن29A. } \\
& .152 \\
& .134 \\
& \begin{array}{cc}
\frac{28072}{9161} & 1028-916 \\
\frac{434}{1462} & .146
\end{array}
\end{aligned}
$$



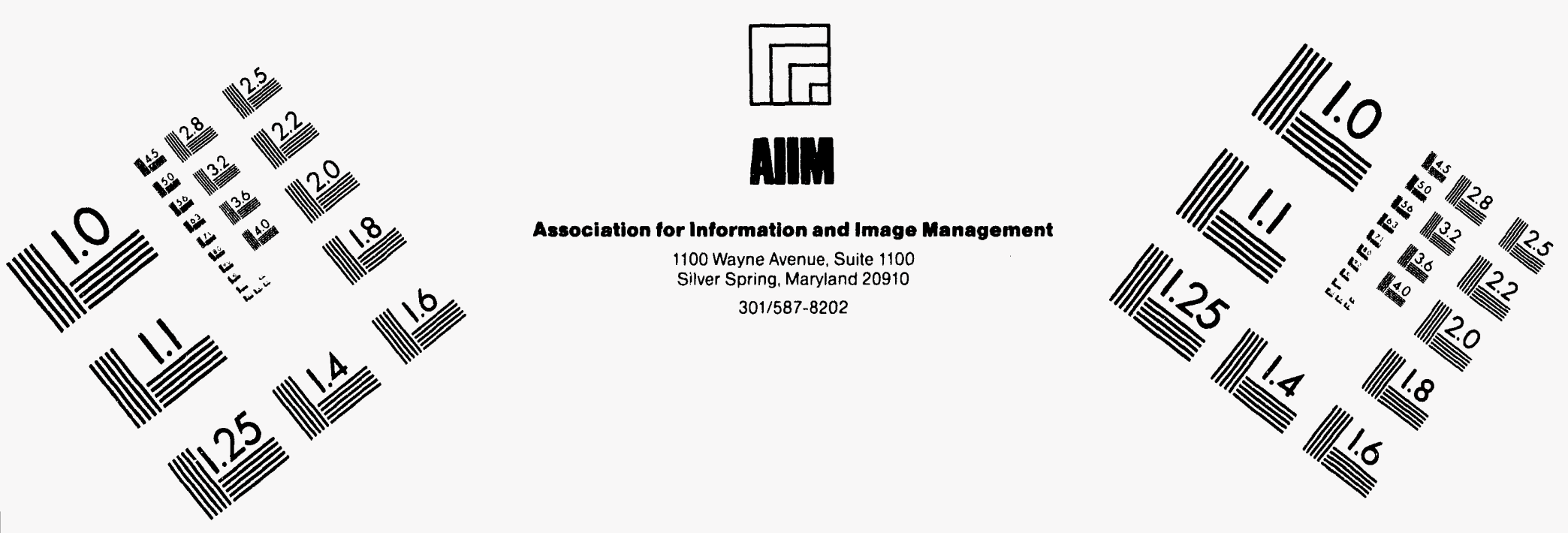

\section{Centimeter}

$\begin{array}{llllllllllllllll}1 & 2 & 3 & 4 & 5 & 6 & 7 & 8 & 9 & 10 & 11 & 12 & 13 & 14 & 15 & \mathrm{~mm}\end{array}$

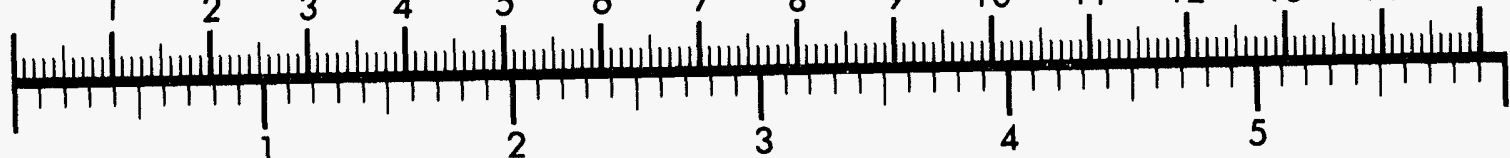
Inches
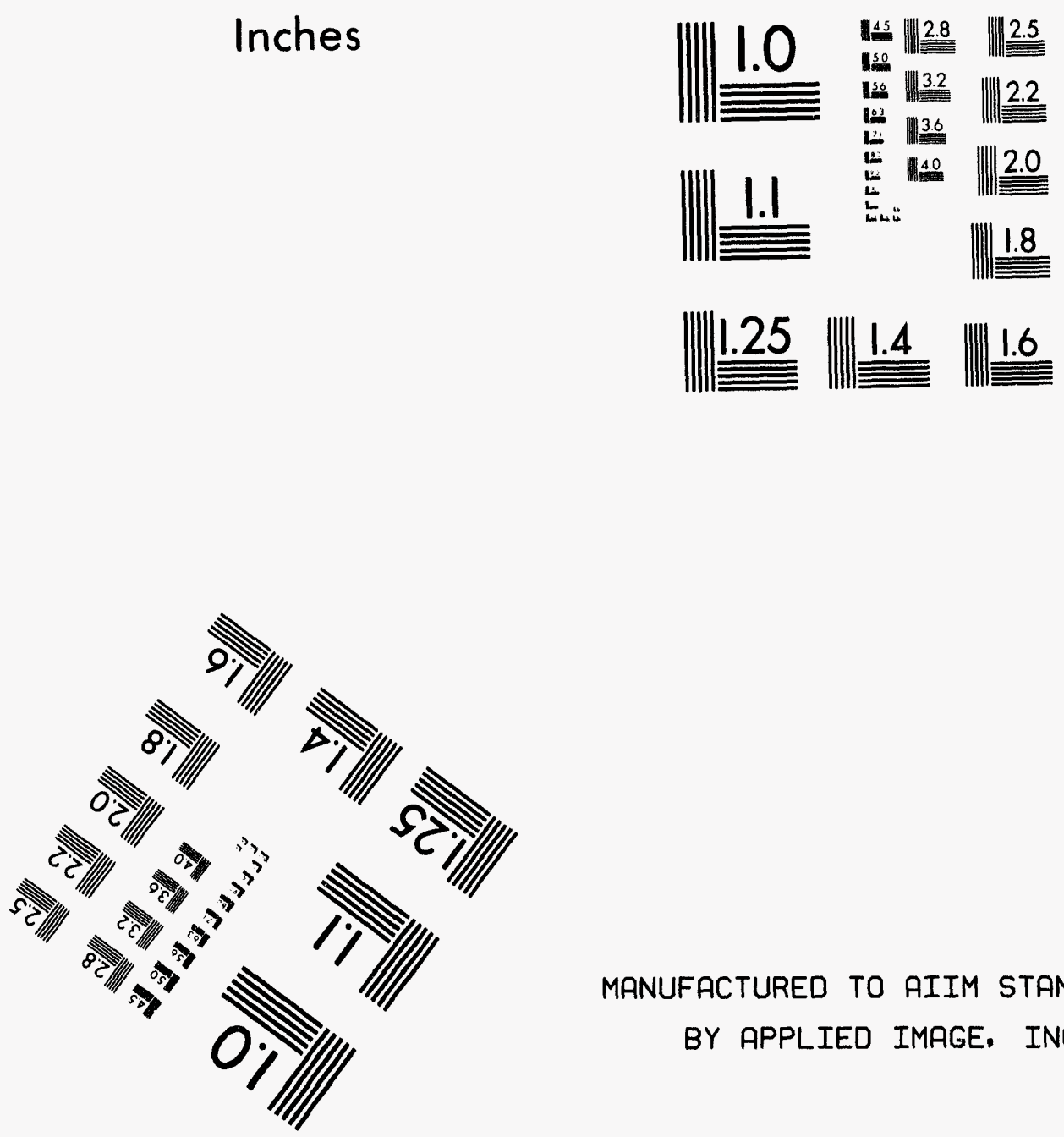

MANUFACTURED TO AIIM STANDARDS

BY APPLIED IMAGE, INC.

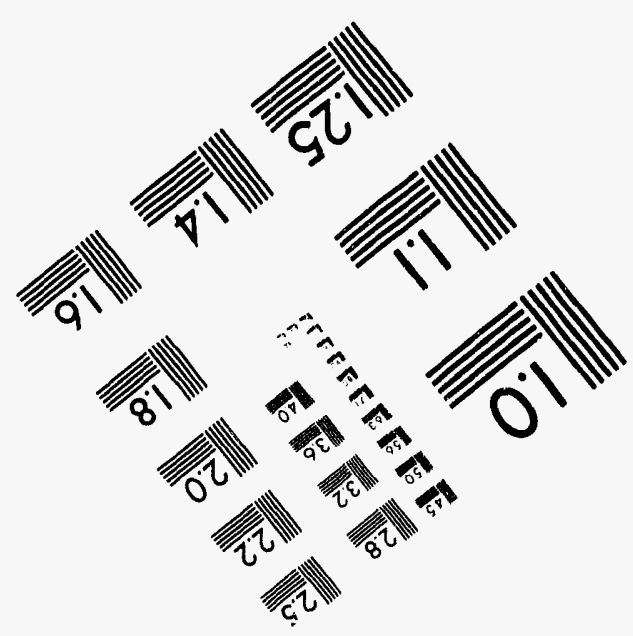



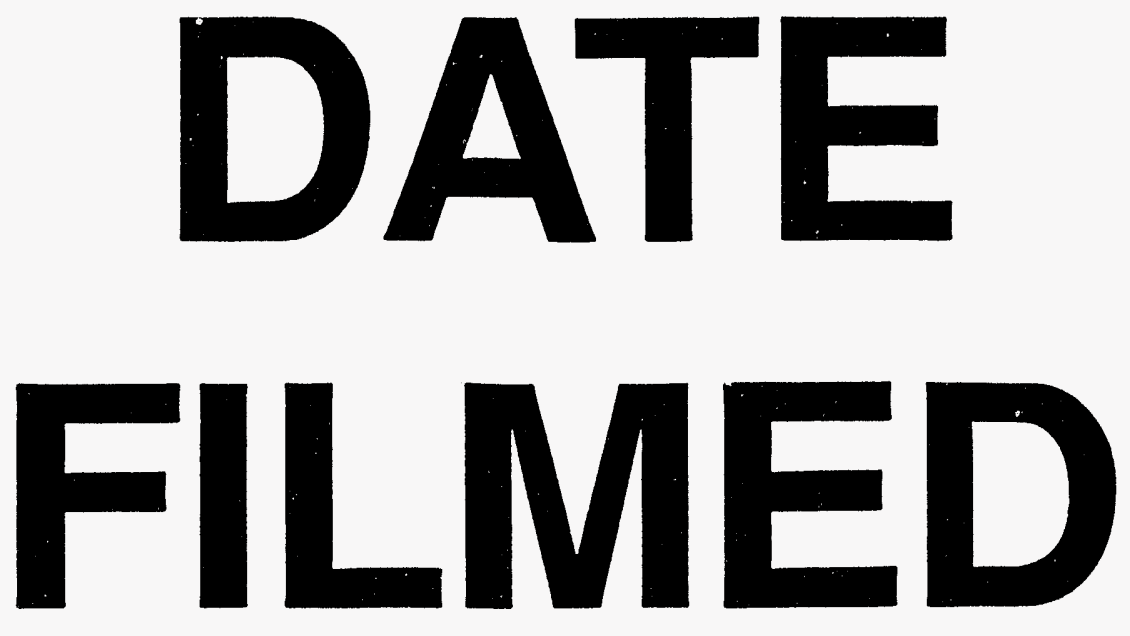

$12 / / 6 / 94$
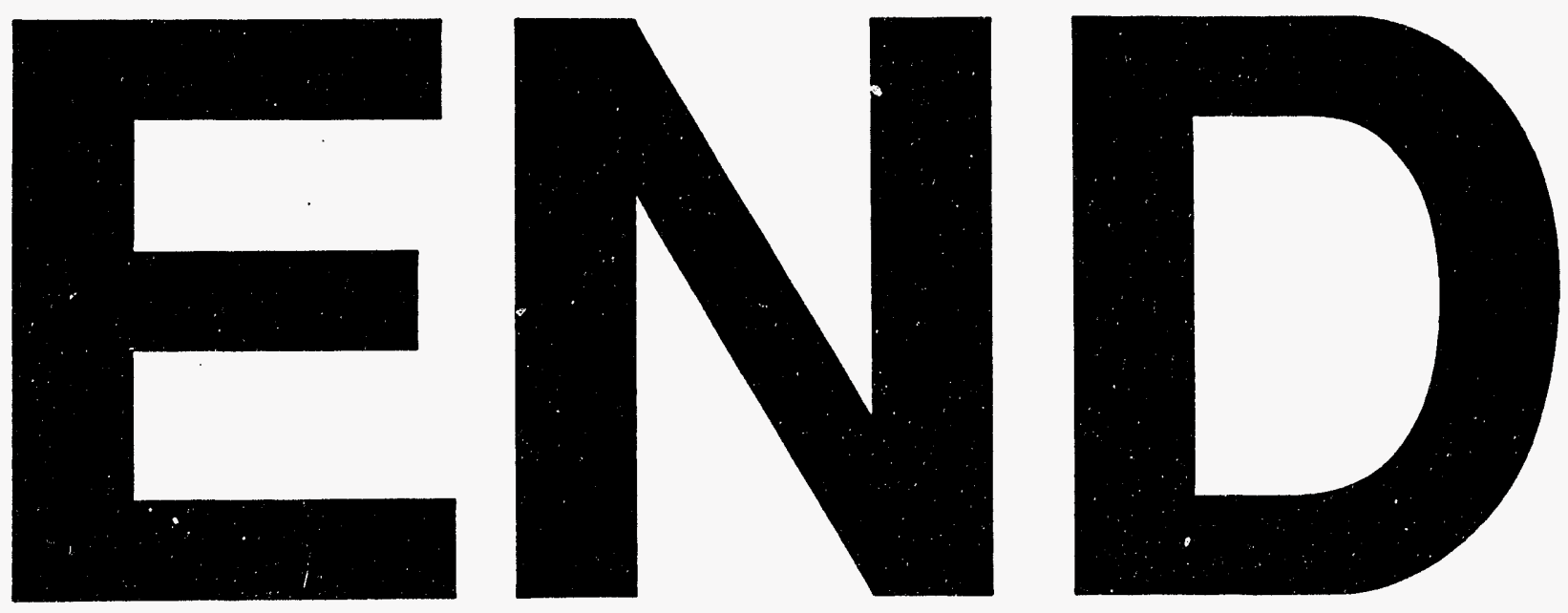